\title{
GLOBAL STRENGTH EVALUATION WITH DESIGN OBLIQUE WAVES CRITERIA FOR A 100000 TDW TANKER
}

\author{
Leonard Domnișoru \\ "Dunarea de Jos" University of Galati, \\ Faculty of Naval Architecture, \\ Galati, Domneasca Street, No. 47, 800008, Romania, \\ E-mail: leonard.domnisoru@ugal.ro
}

\begin{abstract}
Among the preliminary ship's evaluation steps, the global strength of the ship's hull by design oblique wave's criteria is required from the classification society's construction rules. A $100000 \mathrm{tdw}$ tanker is selected for evaluation, with two loading cases. The analysis is applied on a ship hull beam model, using own program software $P Q S W$, with three parameters non-linear equilibrium procedure. Significant oblique design waves are considered, corresponding to maritime operation cases. The global strength results at preliminary design steps are assessed by rules admissible values, so that the ship's global strength design restrictions are obtained.
\end{abstract}

Keywords: global strength, design oblique waves, 100000 tdw tanker.

\section{INTRODUCTION}

In this study the preliminary global strength evaluation of a 100000 tdw tanker [3], [4] is analysed, in the case of design oblique waves. Table 1 presents the main ship's characteristics, with two loading cases, full cargo and ballast. Fig.1 presents the ship's offset lines [4].

Table 1. The 100000 tdw tank data [3], [4]

\begin{tabular}{|c|c|c|c|c|}
\hline$L_{O A}[\mathrm{~m}]$ & 246.0 & Loading: & TK1 cargo & TK2ballast \\
\hline$L_{W L}[\mathrm{~m}]$ & 240.0 & $\Delta[\mathrm{t}]$ & 126457.1 & 81763.3 \\
\hline$B[\mathrm{~m}]$ & 42.0 & $L C G[\mathrm{~m}]$ & 126.46 & 126.73 \\
\hline$H[\mathrm{~m}]$ & 21.3 & $T_{a f f}[\mathrm{~m}]$ & 15.0 & 10.5 \\
\hline$\rho\left[\mathrm{t} / \mathrm{m}^{3}\right]$ & 1.025 & $T_{m}[\mathrm{~m}]$ & 15.0 & 10.0 \\
\hline$g\left[\mathrm{~m} / \mathrm{s}^{2}\right]$ & 9.81 & $T_{\text {fore }}[\mathrm{m}]$ & 15.0 & 9.5 \\
\hline stations & 41 & $\mu[\mathrm{deg}]$ & \multicolumn{2}{|c|}{$0-85$ (step 5) } \\
\hline points & 1230 & $h_{w}[\mathrm{~m}]$ & \multicolumn{2}{|c|}{$0-12$ (step 1) } \\
\hline
\end{tabular}

The global strength analyses for design oblique waves loads [5] are done with own P_QSW program [2], having implemented for wave-ship equilibrium computation a three parameters non-linear procedure (details [2]).
Table 2 presents the admissible sectional efforts from DNV-GL [1] rules for the global strength criteria formulation. This parametric study reveals the tanker global strength limits.

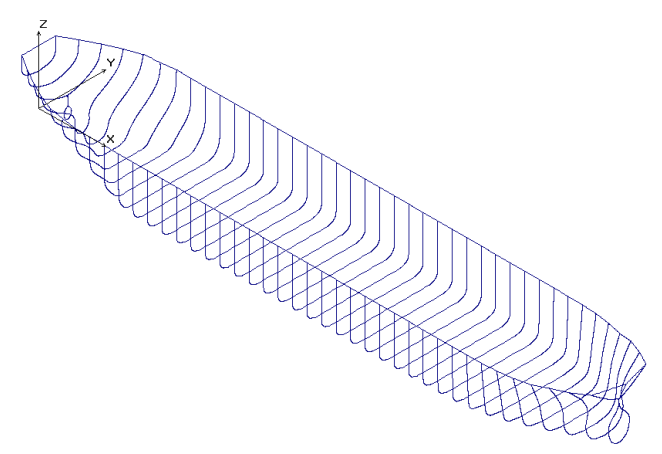

Fig.1.The offset-lines of $100000 \mathrm{tdw}$ tanker [4]

Table 2. Admissible values, DNV-GL [1], vertical, horizontal and torsional efforts

\begin{tabular}{|l|l|}
\hline$V B M_{a d m}[\mathrm{kNm}]$ (bending) & $6.3110^{6}$ \\
\hline$V S F_{a d m}[\mathrm{kN}]$ (shearing) & $9.4310^{4}$ \\
\hline$H B M_{a d m}[\mathrm{kNm}]$ (bending) & $2.2510^{6}$ \\
\hline$H S F_{a d m}[\mathrm{kN}]$ (shearing) & $2.9310^{4}$ \\
\hline$M T_{a d m}[\mathrm{kNm}]$ (torsional) & $7.7010^{5}$ \\
\hline
\end{tabular}




\section{GLOBAL STRENGTH OF 100000 TDW TANKER IN FULL CARGO}

For the 100000 tdw tanker full cargo loading case, the following results on global strength evaluation are obtained:

-Tables 3.a,b present the equilibrium parameters wave-ship (sinkage, pitch and roll) for relevant design oblique waves, full cargo;

-Figs. 2.a,b present the design oblique wave, for $h_{w}=10 \mathrm{~m}, \mu=45$ and $75 \mathrm{deg}$, full cargo case;

-Figs.3.a-e present the efforts diagrams, for oblique wave $\mu=60 \mathrm{deg}$, hogging, full cargo;

-Tables 4.a,b present the maximum efforts, for relevant design oblique waves, full cargo case;

-Figs. 4.a-f present the global strength \& free board criteria evaluation, full cargo case.

Table 3.a Eq. parameters, hogging, full cargo

\begin{tabular}{|c|c|c|c|c|c|}
\hline $\begin{array}{l}\text { Tanker } \\
100000\end{array}$ & $\begin{array}{c}\mu[\mathrm{deg}] \\
h_{w}[\mathrm{~m}] \\
\end{array}$ & 0 & 45 & 60 & 75 \\
\hline \multirow{4}{*}{$\begin{array}{c}T_{m} \\
{[\mathrm{~m}]}\end{array}$} & 0 & 15.000 & 15.000 & 15.000 & 15.000 \\
\hline & 5 & 14.680 & 14.756 & 14.895 & 15.359 \\
\hline & 10 & 14.266 & 14.427 & 14.721 & 15.699 \\
\hline & 12 & 14.082 & 14.277 & 14.632 & 15.833 \\
\hline \multirow{4}{*}{$\theta[\mathrm{rad}]$} & 0 & & 0.0000 & 0.0 & 0.0000 \\
\hline & 5 & 0.0011 & 0.0009 & 0.0005 & -0.0007 \\
\hline & 10 & & 0.0040 & 0.0027 & -0.0010 \\
\hline & 12 & 0.0067 & 0.0058 & 0.0041 & -0.0011 \\
\hline \multirow{4}{*}{$\varphi[\mathrm{rad}]$} & U & 0.0000 & 0.0000 & 0.0000 & 0.0000 \\
\hline & 5 & 0.0000 & 0.0008 & 0.0010 & 0.0003 \\
\hline & 10 & 0.0000 & 0.0029 & 0.0037 & 0.0005 \\
\hline & 12 & 0.0000 & 0.0042 & 0.0053 & 0.0006 \\
\hline
\end{tabular}

Table 3.b Eq. parameters, sagging, full cargo

\begin{tabular}{|c|c|c|c|c|c|}
\hline $\begin{array}{c}\text { Tanker } \\
100000\end{array}$ & $h_{w}[\mathrm{deg}]$ & 0 & 45 & 60 & 75 \\
\hline \multirow{4}{*}{$\begin{array}{c}T_{m} \\
{[\mathrm{~m}]}\end{array}$} & 0 & 15.000 & 15.000 & 15.000 & 15.000 \\
\cline { 2 - 6 } & 5 & 15.243 & 15.180 & 15.064 & 14.627 \\
\cline { 2 - 6 } & 10 & 15.442 & 15.327 & 15.108 & 14.227 \\
\cline { 2 - 6 } & 12 & 15.511 & 15.380 & 15.122 & 14.060 \\
\hline \multirow{4}{*}{$\theta[\mathrm{rad}]$} & 0 & 0.0000 & 0.0000 & 0.0000 & 0.0000 \\
\cline { 2 - 6 } & 5 & 0.0001 & 0.0001 & 0.0002 & 0.0009 \\
\cline { 2 - 6 } & 10 & -0.0002 & 0.0000 & 0.0003 & 0.0022 \\
\cline { 2 - 6 } & 12 & -0.0004 & -0.0001 & 0.0003 & 0.0029 \\
\hline \multirow{4}{*}{$\varphi[\mathrm{rad}]$} & 0 & 0.0000 & 0.0000 & 0.0000 & 0.0000 \\
\cline { 2 - 6 } & 5 & 0.0000 & -0.0001 & -0.0003 & -0.0011 \\
\cline { 2 - 6 } & 10 & 0.0000 & -0.0003 & -0.0005 & -0.0035 \\
\cline { 2 - 6 } & 12 & 0.0000 & -0.0004 & -0.0007 & -0.0049 \\
\hline
\end{tabular}

62

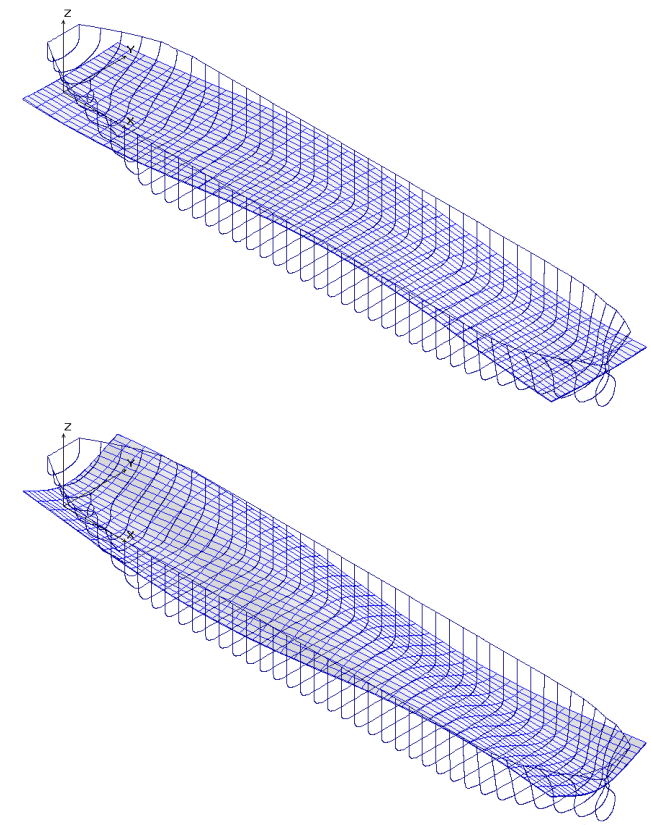

Fig.2.a Hogging design oblique wave, $h_{w}=10 \mathrm{~m}, \mu=45$ and $75 \mathrm{deg}, 100000 \mathrm{tdw}$ tanker, full cargo loading case
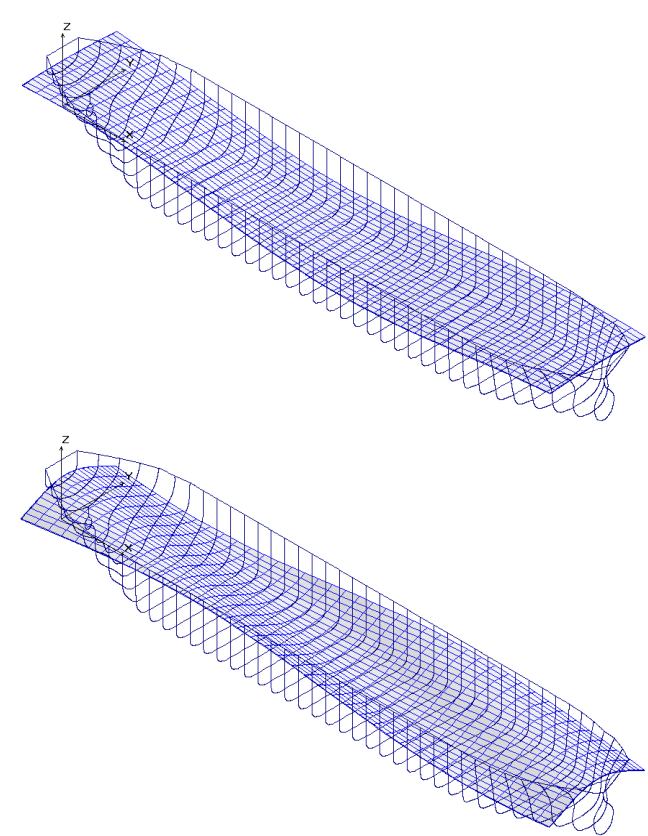

Fig.2.b Sagging design oblique wave, $h_{w}=10 \mathrm{~m}, \mu=45$ and $75 \mathrm{deg}, 100000 \mathrm{tdw}$ tanker, full cargo loading case

(C) Galati University Press, 2020 


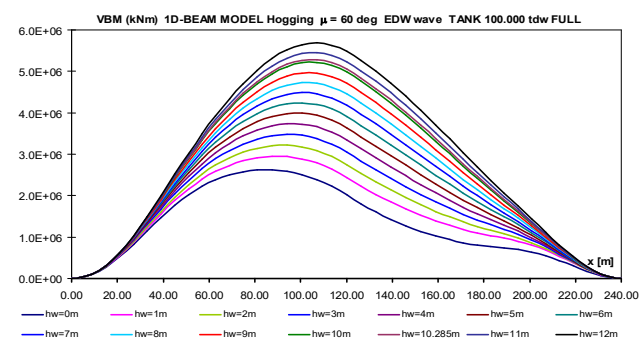

Fig.3.a $V B M[\mathrm{kNm}], \mu=60 \mathrm{deg}$, hogg, full cargo

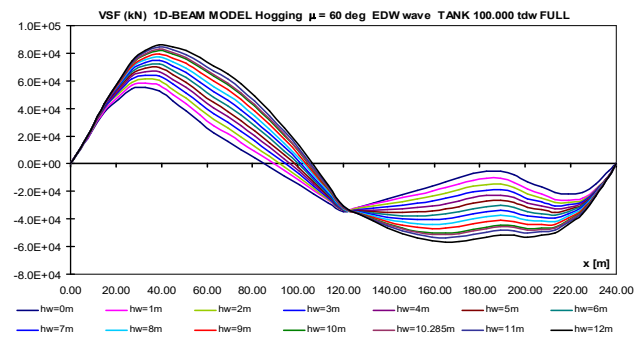

Fig.3.b $\operatorname{VSF}[\mathrm{kN}], \mu=60 \mathrm{deg}$, hogg, full cargo

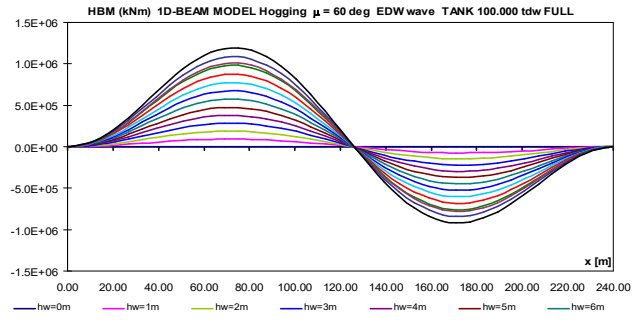

Fig.3.c $H B M[\mathrm{kNm}], \mu=60 \mathrm{deg}$, hogg, full cargo

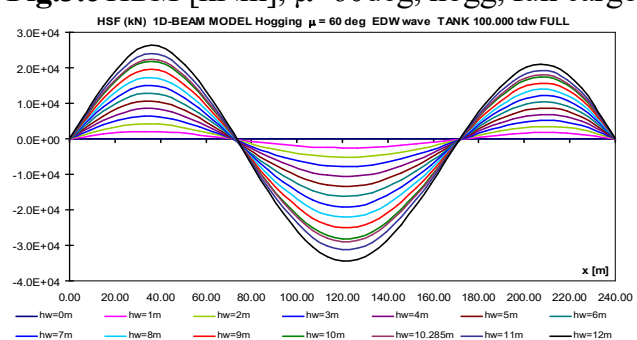

Fig.3.d $H S F[\mathrm{kN}], \mu=60 \mathrm{deg}$, hogg, full cargo

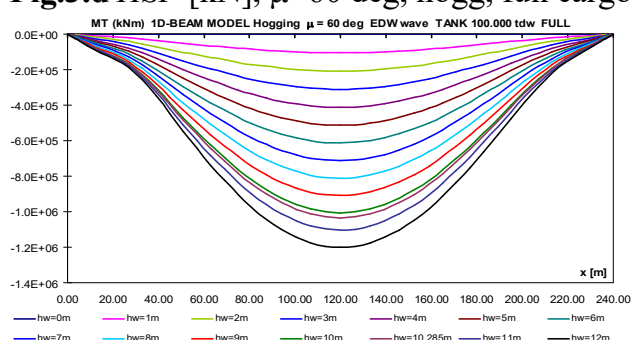

Fig.3.e $M T[\mathrm{kNm}], \mu=60 \mathrm{deg}$, hogg, full cargo

(C) Galati University Press, 2020
Table 4.a Max. efforts, hogging, full cargo

\begin{tabular}{|c|c|c|c|c|c|}
\hline Tar & $\begin{array}{l}\mathrm{eg}] \\
\mathrm{m}]\end{array}$ & 0 & 45 & 60 & 75 \\
\hline \multirow{4}{*}{$\begin{array}{c}V B M \\
{[\mathrm{kNm}]}\end{array}$} & 0 & $2.610^{6}$ & $2.610^{6}$ & $2.610^{6}$ & 2.610 \\
\hline & 5 & $4.710^{6}$ & $4.410^{6}$ & $4.010^{6}$ & 2.410 \\
\hline & 10 & $6.610^{6}$ & $6.110^{6}$ & 5.21 & $2.110^{6}$ \\
\hline & 12 & $7.310^{6}$ & 6.8 & 5.7 & $2.010^{6}$ \\
\hline \multirow{4}{*}{$\begin{array}{l}V S F \\
{[\mathrm{kN}]}\end{array}$} & 0 & 5.5 & 5.5 & 5.5 & \begin{tabular}{|lll}
5.5 & $10^{4}$ \\
\end{tabular} \\
\hline & 5 & $7.610^{4}$ & $7.410^{4}$ & $7.010^{4}$ & \begin{tabular}{|lll}
5.6 & $10^{4}$ \\
\end{tabular} \\
\hline & 10 & $9.510^{4}$ & $9.010^{4}$ & $8.210^{4}$ & $5.610^{4}$ \\
\hline & 12 & $1.010^{5}$ & $9.710^{4}$ & $8.610^{4}$ & $5.610^{4}$ \\
\hline \multirow{4}{*}{$\begin{array}{c}H B M \\
{[\mathrm{kNm}]}\end{array}$} & 0 & 0.0 & 0.0 & 0.0 & 0.0 \\
\hline & 5 & 0.0 & $3.1 \quad 10^{5}$ & $4.710^{5}$ & \begin{tabular}{|lll}
5.0 & $10^{5}$ \\
\end{tabular} \\
\hline & 10 & 0.0 & $6.410^{5}$ & $9.810^{5}$ & $1.010^{6}$ \\
\hline & 12 & 0.0 & $7.710^{5}$ & $1.210^{6}$ & $1.210^{6}$ \\
\hline \multirow{4}{*}{$\begin{array}{l}H S F \\
{[\mathrm{kN}]}\end{array}$} & 0 & & 0.0 & 0.0 & 0.0 \\
\hline & 5 & 0.0 & $8.810^{3}$ & $1.310^{4}$ & $1.310^{4}$ \\
\hline & 10 & 0.0 & $1.910^{4}$ & $2.810^{4}$ & $2.610^{4}$ \\
\hline & 12 & 0.0 & $2.310^{4}$ & $3.410^{4}$ & $3.110^{4}$ \\
\hline \multirow{4}{*}{$\begin{array}{c}M T \\
{[\mathrm{kNm}]}\end{array}$} & U & 0.0 & 0.0 & 0.0 & 0.0 \\
\hline & 5 & 0.0 & $3.210^{5}$ & $5.110^{5}$ & $6.610^{\circ}$ \\
\hline & 10 & 0.0 & $6.310^{5}$ & $1.010^{6}$ & $1.310^{6}$ \\
\hline & 12 & 0.0 & $7.410^{5}$ & $1.310^{6}$ & 1.610 \\
\hline
\end{tabular}

Table 4.b Max. efforts, sagging, full cargo

\begin{tabular}{|c|c|c|c|c|c|}
\hline $\begin{array}{l}\text { Tanl } \\
1000\end{array}$ & $\mathrm{~g}$ & 0 & 45 & 60 & 75 \\
\hline \multirow{4}{*}{$\begin{array}{c}V B M \\
{[\mathrm{kNm}]}\end{array}$} & 0 & $2.610^{6}$ & $2.610^{6}$ & $2.610^{6}$ & $2.610^{6}$ \\
\hline & 5 & $4.710^{6}$ & 1.4 & 1.6 & $3.110^{6}$ \\
\hline & 10 & $6.610^{6}$ & $2.510^{6}$ & $1.510^{6}$ & $3.510^{6}$ \\
\hline & 12 & 7.3 & 3.4 & 2.1 & $3.610^{6}$ \\
\hline \multirow{4}{*}{$\begin{array}{l}V S F \\
{[\mathrm{kN}]}\end{array}$} & 0 & 5.5 & $5.510^{4}$ & $5.510^{4}$ & $5.510^{4}$ \\
\hline & 5 & 7.6 & 3.9 & 4.2 & $5.610^{4}$ \\
\hline & 10 & $9.510^{4}$ & $5.410^{4}$ & $3.7 \quad 10^{4}$ & $5.710^{4}$ \\
\hline & 12 & $1.010^{5}$ & $6.610^{4}$ & $4.610^{4}$ & $5.710^{4}$ \\
\hline \multirow{4}{*}{$\begin{array}{c}H B M \\
{[\mathrm{kNm}]}\end{array}$} & 0 & 0.0 & 0.0 & 0.0 & 0.0 \\
\hline & 5 & 0.0 & $2.810^{5}$ & $4.410^{5}$ & $5.010^{5}$ \\
\hline & 10 & 0.0 & $5.410^{5}$ & $8.510^{5}$ & $9.910^{5}$ \\
\hline & 12 & 0.0 & $6.310^{5}$ & $1.010^{6}$ & $1.210^{6}$ \\
\hline \multirow{4}{*}{$\begin{array}{l}H S F \\
{[\mathrm{kN}]}\end{array}$} & & 0. & 0.0 & 0.0 & 0.0 \\
\hline & 5 & 0.0 & $7.410^{3}$ & $1.210^{4}$ & $1.410^{4}$ \\
\hline & 10 & 0.0 & $1.310^{4}$ & $2.210^{4}$ & $2.810^{4}$ \\
\hline & 12 & 0.0 & $1.510^{4}$ & $2.610^{4}$ & $3.410^{4}$ \\
\hline \multirow{4}{*}{$\begin{array}{c}M T \\
{[\mathrm{kNm}]}\end{array}$} & 0 & 0.0 & 0.0 & 0.0 & 0.0 \\
\hline & 5 & 0.0 & $3.510^{5}$ & $5.410^{5}$ & $6.610^{5}$ \\
\hline & 10 & 0.0 & $7.310^{5}$ & $1.110^{6}$ & $1.310^{6}$ \\
\hline & 12 & 0.0 & $8.810^{5}$ & $1.410^{6}$ & $1.610^{6}$ \\
\hline
\end{tabular}




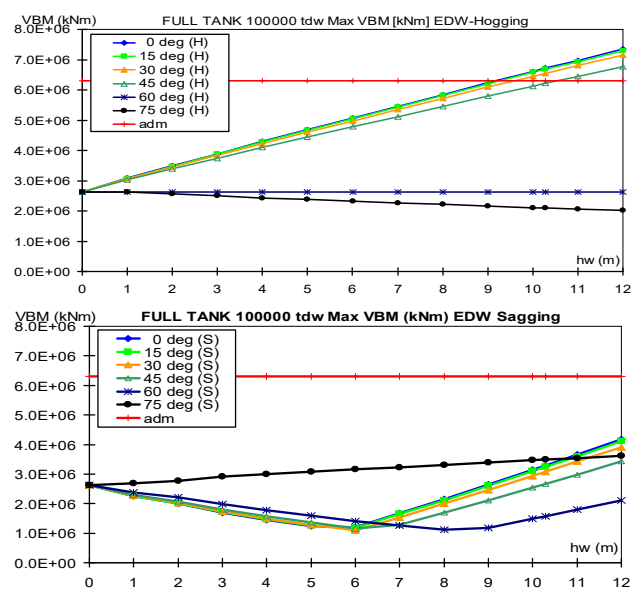

Fig.4.a $V B M_{\max }[\mathrm{kNm}]$, TK 100000, full cargo
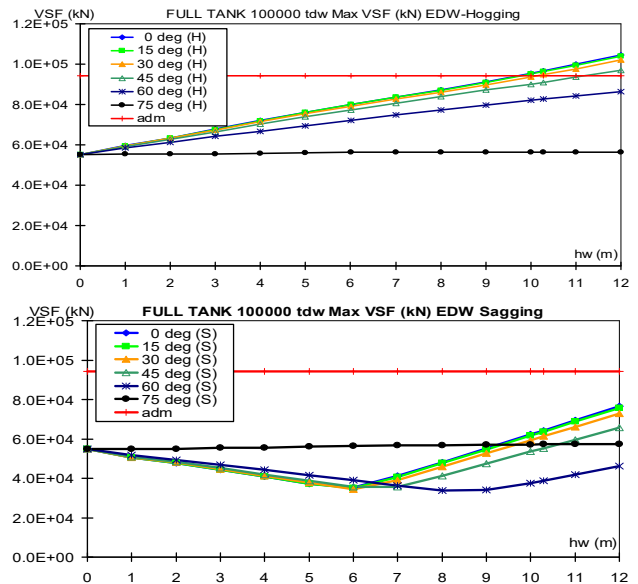

Fig.4.b $V S F_{\max }[\mathrm{kN}]$, TK 100000, full cargo
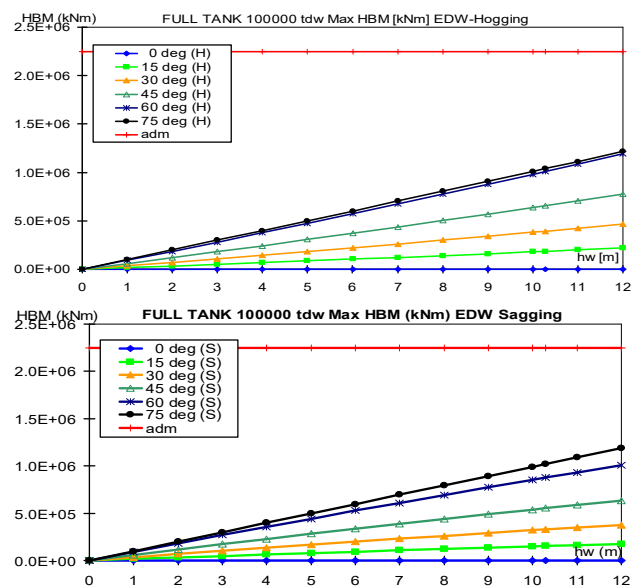

Fig.4.c $H B M_{\max }[\mathrm{kNm}]$, TK 100000, full cargo 64
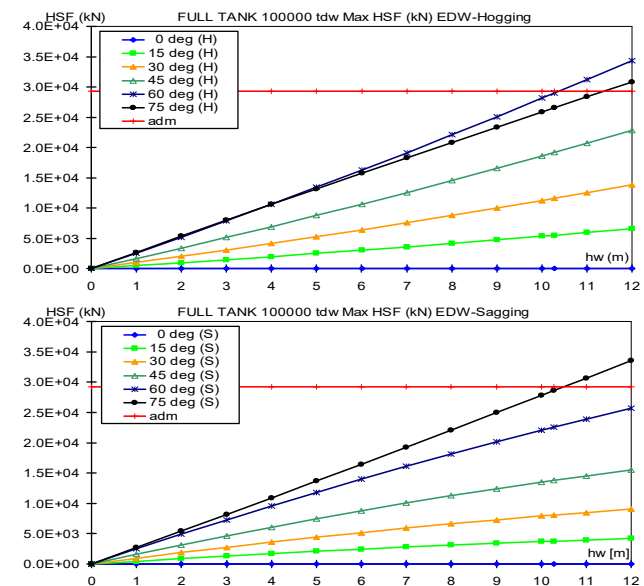

Fig.4.d $H S F_{\max }[\mathrm{kN}]$, TK 100000, full cargo

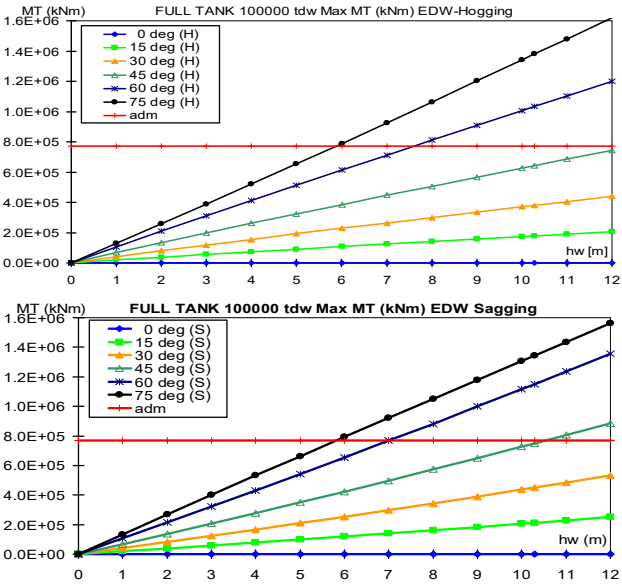

Fig.4.e $M T_{\max }[\mathrm{kNm}]$, TK 100000, full cargo Zaft_PS ( $m$ ) FULL TANK 100000 tdw Max Zaft_PS ( $m$ ) EDW-Hogg \& Sagg

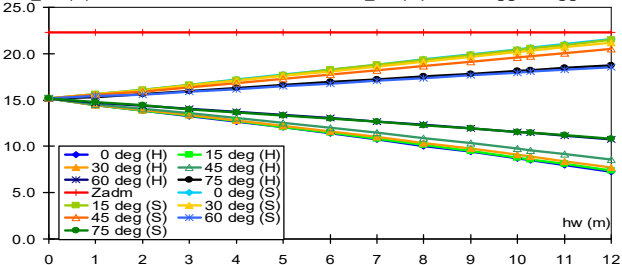

Zfore_PS (m) FULL TANK 100000 tdw Max Zfore_PS (m) EDW-Hogg \& Sagg

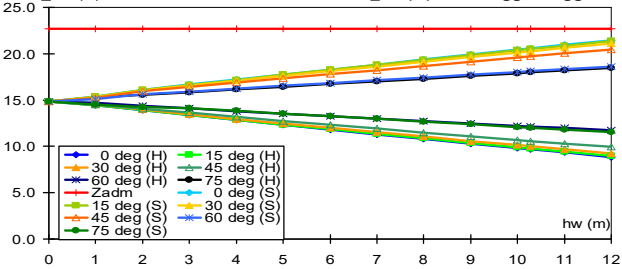

Fig.4.f $Z_{\max }[\mathrm{m}]$, aft-fore, TK 100000, full cargo

(C) Galati University Press, 2020 


\section{GLOBAL STRENGTH OF 100000 TDW TANKER IN BALLAST}

For the 100000 tdw tanker ballast loading case, the following results on global strength evaluation are obtained:

-Tables 5.a,b present the equilibrium parameters wave-ship (sinkage, pitch and roll) for relevant design oblique waves, ballast case;

-Figs. 5.a,b present the design oblique wave, for $h_{w}=10 \mathrm{~m}, \mu=45$ and $75 \mathrm{deg}$, ballast case;

-Figs. 6.a-e present the efforts diagrams, for oblique wave $\mu=60 \mathrm{deg}$, sagging, ballast case; -Tables 6.a,b present the maximum efforts, for relevant design oblique waves, ballast case; -Figs. 7.a-f present the global strength \& free board criteria evaluation, ballast case.

Table 5.a Eq. parameters, hogging, ballast

\begin{tabular}{|c|c|c|c|c|c|}
\hline $\begin{array}{l}\text { Tanker } \\
100000\end{array}$ & $\begin{array}{c}\mu[\mathrm{deg}] \\
h_{w}[\mathrm{~m}] \\
\end{array}$ & 0 & 45 & 60 & 75 \\
\hline \multirow{4}{*}{$\begin{array}{c}T_{m} \\
{[\mathrm{~m}]}\end{array}$} & 0 & 10.000 & 10.000 & 10.000 & 10.000 \\
\hline & 5 & 9.547 & 9.639 & 9.809 & 10.421 \\
\hline & 10 & 9.038 & 9.222 & 9.563 & 10.799 \\
\hline & 12 & 8.826 & 9.048 & 9.457 & 10.947 \\
\hline \multirow{4}{*}{$\theta[\mathrm{rad}]$} & 0 & -0.0043 & -0.0043 & -0.0043 & -0.0043 \\
\hline & 5 & 0.0007 & 0.0002 & -0.0009 & -0.0049 \\
\hline & 10 & 0.0074 & 0.0062 & 0.0041 & -0.0044 \\
\hline & 12 & 0.0102 & 0.0088 & 0.0062 & -0.0042 \\
\hline \multirow{4}{*}{$\varphi[\mathrm{rad}]$} & 0 & 0.0000 & 0.0000 & 0.0000 & 0.0000 \\
\hline & 5 & 0.0000 & 0.0024 & 0.0034 & 0.0023 \\
\hline & 10 & 0.0000 & 0.0065 & 0.0090 & 0.0038 \\
\hline & 12 & 0.0000 & 0.0085 & 0.0118 & 0.0042 \\
\hline
\end{tabular}

Table 5.b Eq. parameters, sagging, ballast

\begin{tabular}{|c|c|c|c|c|c|}
\hline $\begin{array}{c}\text { Tanker } \\
100000\end{array}$ & $h_{w}[\mathrm{deg}]$ & 0 & 45 & 60 & 75 \\
\hline \multirow{4}{*}{$\begin{array}{c}T_{m} \\
{[\mathrm{~m}]}\end{array}$} & 0 & 10.000 & 10.000 & 10.000 & 10.000 \\
\cline { 2 - 6 } & 5 & 10.387 & 10.303 & 10.145 & 9.557 \\
\cline { 2 - 6 } & 10 & 10.637 & 10.526 & 10.230 & 9.080 \\
\cline { 2 - 6 } & 12 & 10.782 & 10.554 & 10.209 & 8.845 \\
\hline \multirow{5}{*}{$\theta[\mathrm{rad}]$} & 0 & -0.0043 & -0.0043 & -0.0043 & -0.0043 \\
\cline { 2 - 6 } & 5 & -0.0070 & -0.0068 & -0.0062 & -0.0031 \\
\cline { 2 - 6 } & 10 & -0.0072 & -0.0069 & -0.0062 & -0.0009 \\
\cline { 2 - 6 } & 12 & -0.0069 & -0.0081 & -0.0073 & -0.0002 \\
\hline \multirow{4}{*}{$\varphi[\mathrm{rad}]$} & 0 & 0.0000 & 0.0000 & 0.0000 & 0.0000 \\
\cline { 2 - 6 } & 5 & 0.0000 & -0.0008 & -0.0016 & -0.0037 \\
\cline { 2 - 6 } & 10 & 0.0000 & -0.0005 & -0.0023 & -0.0090 \\
\cline { 2 - 6 } & 12 & 0.0000 & -0.0002 & -0.0023 & -0.0116 \\
\hline
\end{tabular}

(C) Galati University Press, 2020
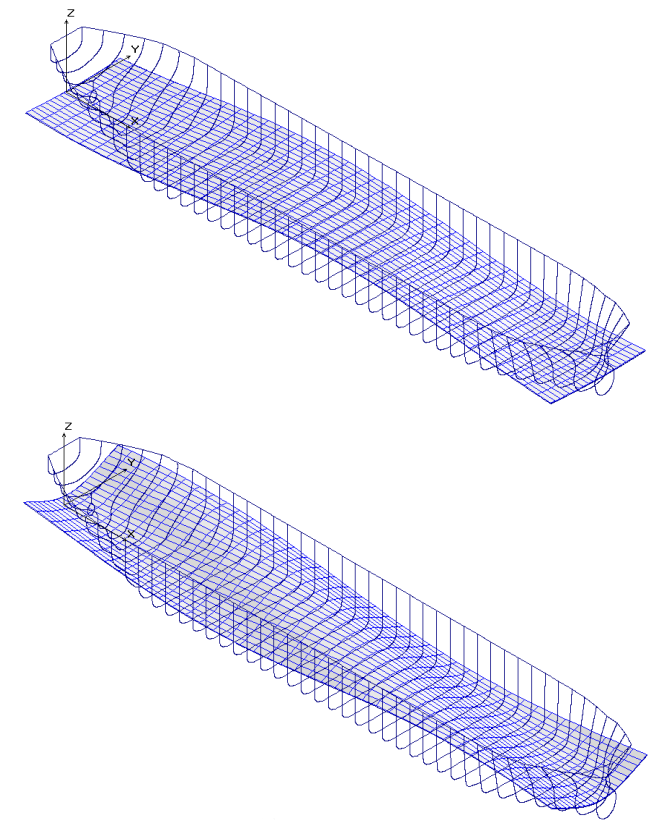

Fig.5.a Hogging design oblique wave, $h_{w}=10 \mathrm{~m}, \mu=45$ and $75 \mathrm{deg}, 100000 \mathrm{tdw}$ tanker, ballast loading case
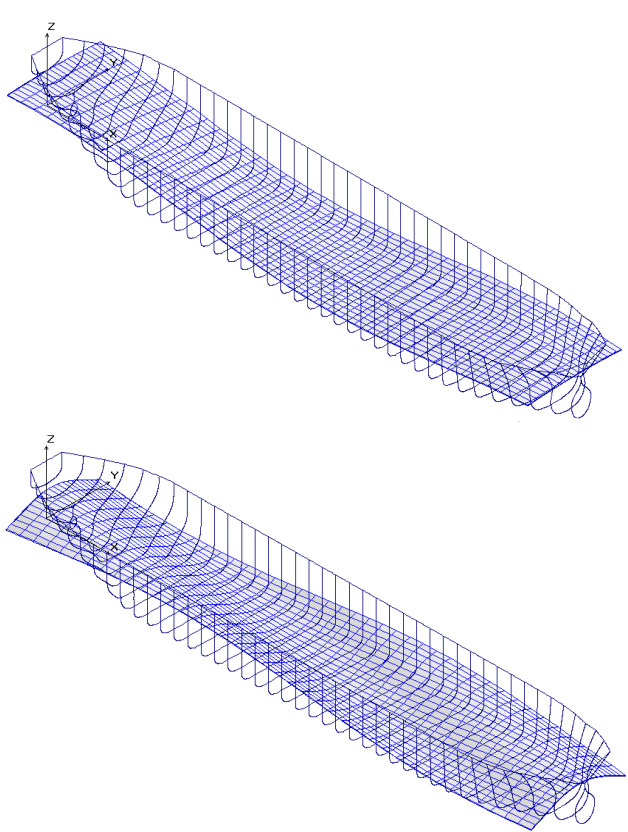

Fig.5.b Sagging design oblique wave, $h_{w}=10 \mathrm{~m}, \mu=45$ and $75 \mathrm{deg}, 100000 \mathrm{tdw}$ tanker, ballast loading case 


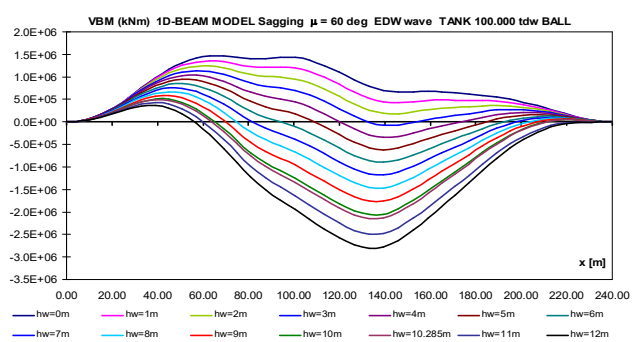

Fig.6.a $V B M[\mathrm{kNm}], \mu=60 \mathrm{deg}$, sagg, ballast
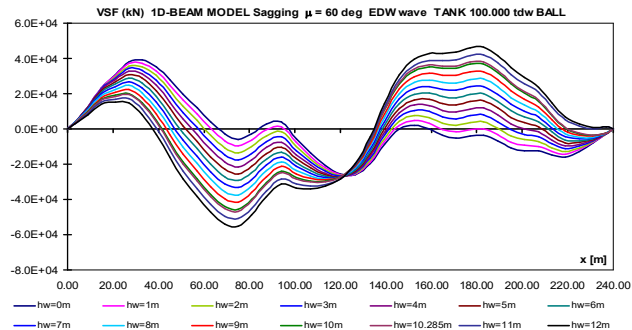

Fig.6.b $\operatorname{VSF}[\mathrm{kN}], \mu=60 \mathrm{deg}$, sagg, ballast

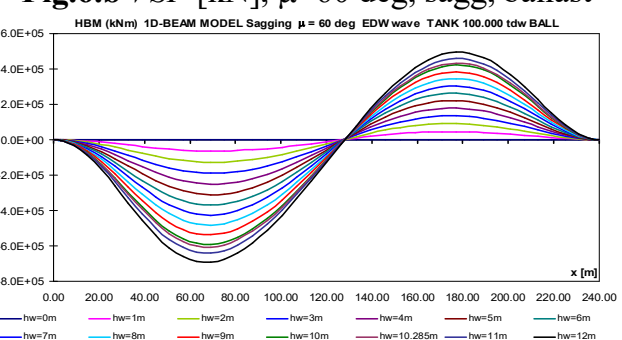

Fig.6.c $H B M[\mathrm{kNm}], \mu=60 \mathrm{deg}$, sagg, ballast

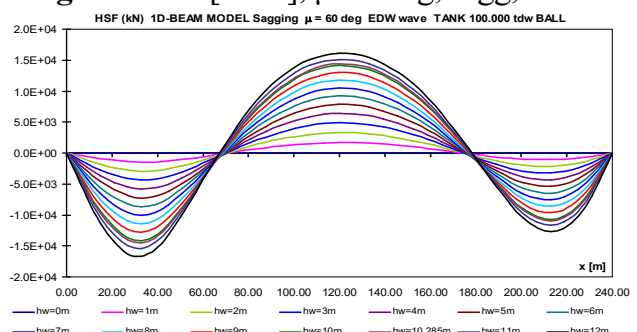

Fig.6.d $H S F[\mathrm{kN}], \mu=60 \mathrm{deg}$, sagg, ballast

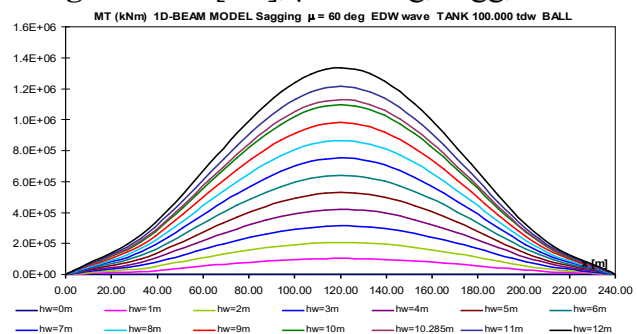

Fig.6.e $M T[\mathrm{kNm}], \mu=60 \mathrm{deg}$, sagg, ballast 66
Table 6.a Max. efforts, hogging, ballast

\begin{tabular}{|c|c|c|c|c|c|}
\hline $\begin{array}{c}\text { Tanker } \\
100000\end{array}$ & {$[\mathrm{~m}]$} & 0 & 45 & 60 & 75 \\
\hline \multirow{4}{*}{$\begin{array}{c}V B M \\
{[\mathrm{kNm}]}\end{array}$} & 0 & $1.410^{6}$ & $1.410^{6}$ & $1.410^{6}$ & $1.410^{6}$ \\
\hline & 5 & $3.210^{6}$ & 3.0 & $2.610^{6}$ & $1.410^{6}$ \\
\hline & 10 & $4.910^{6}$ & 4.4 & 3.6 & $1.310^{6}$ \\
\hline & 12 & 5.6 & 5.0 & 4.1 & $1.210^{6}$ \\
\hline \multirow{4}{*}{$\begin{array}{l}V S F \\
{[\mathrm{kN}]}\end{array}$} & 0 & $3.910^{4}$ & 3.9 & $3.910^{4}$ & $3.910^{4}$ \\
\hline & 5 & $5.010^{4}$ & $4.910^{4}$ & $4.710^{4}$ & $4.010^{4}$ \\
\hline & 10 & $6.410^{4}$ & $6.010^{4}$ & $5.410^{4}$ & $3.910^{4}$ \\
\hline & 12 & $7.310^{4}$ & $6.610^{4}$ & $5.810^{4}$ & $3.910^{4}$ \\
\hline \multirow{4}{*}{$\begin{array}{c}H B M \\
{[\mathrm{kNm}]}\end{array}$} & & 0.0 & 0.0 & 0.0 & 0.0 \\
\hline & 5 & 0.0 & $2.110^{5}$ & $3.310^{5}$ & $3.610^{5}$ \\
\hline & 10 & 0.0 & 4.4 & 6.9 & 7.4 \\
\hline & 12 & 0.0 & $5.410^{5}$ & $8.310^{5}$ & $8.910^{5}$ \\
\hline \multirow{4}{*}{$\begin{array}{l}H S F \\
{[\mathrm{kN}]}\end{array}$} & 0 & 0.0 & 0.0 & 0.0 & 0.0 \\
\hline & 5 & 0.0 & $6.010^{3}$ & $9.110^{3}$ & $9.010^{3}$ \\
\hline & 10 & 0.0 & $1.310^{4}$ & $1.910^{4}$ & $1.810^{4}$ \\
\hline & 12 & 0.0 & $1.610^{4}$ & $2.410^{4}$ & $2.210^{4}$ \\
\hline \multirow{4}{*}{$\begin{array}{c}M T \\
{[\mathrm{kNm}]}\end{array}$} & 0 & 0.0 & 0.0 & 0.0 & 0.0 \\
\hline & (3 & 0.0 & $3.110^{5}$ & $5.010^{5}$ & $6.410^{5}$ \\
\hline & 10 & 0.0 & $6.010^{5}$ & $9.710^{5}$ & $1.310^{6}$ \\
\hline & 12 & 0.0 & $7.110^{5}$ & $1.210^{6}$ & $1.610^{6}$ \\
\hline
\end{tabular}

Table 6.b Max. efforts, sagging, ballast

\begin{tabular}{|c|c|c|c|c|c|}
\hline $\begin{array}{l}\text { Tanl } \\
1000\end{array}$ & $\mathrm{w}[\mathrm{m}]$ & 0 & 45 & 60 & 75 \\
\hline \multirow{4}{*}{$\begin{array}{c}V B M \\
{[\mathrm{kNm}]}\end{array}$} & 0 & $1.410^{6}$ & $1.410^{6}$ & $1.410^{6}$ & $1.410^{6}$ \\
\hline & 5 & 1.3 & 1.1 & $9.410^{5}$ & $1.810^{6}$ \\
\hline & 10 & $3.610^{6}$ & 3.0 & $2.110^{6}$ & $2.110^{6}$ \\
\hline & 12 & 4.5 & 4.0 & 2.8 & $2.210^{6}$ \\
\hline \multirow{4}{*}{$\begin{array}{c}V S F \\
{[\mathrm{kN}]}\end{array}$} & 0 & $3.910^{4}$ & 3.9 & $3.910^{4}$ & $3.910^{4}$ \\
\hline & 5 & $3.710^{4}$ & $3.310^{4}$ & $3.110^{4}$ & $3.710^{4}$ \\
\hline & 10 & $6.910^{4}$ & 6.0 & 4.6 & $3.510^{4}$ \\
\hline & 12 & $8.110^{4}$ & $7.310^{4}$ & $5.510^{4}$ & $3.310^{4}$ \\
\hline \multirow{4}{*}{$\begin{array}{c}H B M \\
{[\mathrm{kNm}]}\end{array}$} & & 0.0 & 0.0 & 00 & 0.0 \\
\hline & 5 & 0.0 & $2.010^{5}$ & $3.110^{5}$ & $3.510^{5}$ \\
\hline & 10 & 0.0 & $3.710^{5}$ & $5.910^{5}$ & $6.910^{5}$ \\
\hline & 12 & 0.0 & $4.410^{5}$ & $6.910^{5}$ & $8.210^{5}$ \\
\hline \multirow{4}{*}{$\begin{array}{l}H S F \\
{[\mathrm{kN}]}\end{array}$} & ( & 0.0 & 0.0 & 0.0 & 0.0 \\
\hline & 5 & 0.0 & $4.910^{3}$ & $7.810^{3}$ & $9.310^{3}$ \\
\hline & 10 & 0.0 & $9.110^{3}$ & $1.410^{4}$ & $1.910^{4}$ \\
\hline & 12 & 0.0 & $1.110^{4}$ & $1.710^{4}$ & $2.310^{4}$ \\
\hline \multirow{4}{*}{$\begin{array}{c}M T \\
{[\mathrm{kNm}]}\end{array}$} & 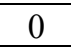 & 0.0 & 0.0 & 0.0 & 0.0 \\
\hline & כ & 0.0 & $3.410^{5}$ & $5.310^{5}$ & $6.510^{5}$ \\
\hline & 10 & 0.0 & $7.210^{5}$ & $1.110^{6}$ & $1.310^{6}$ \\
\hline & 12 & 0.0 & $8.810^{5}$ & $1.310^{6}$ & 1.510 \\
\hline
\end{tabular}

(C) Galati University Press, 2020 

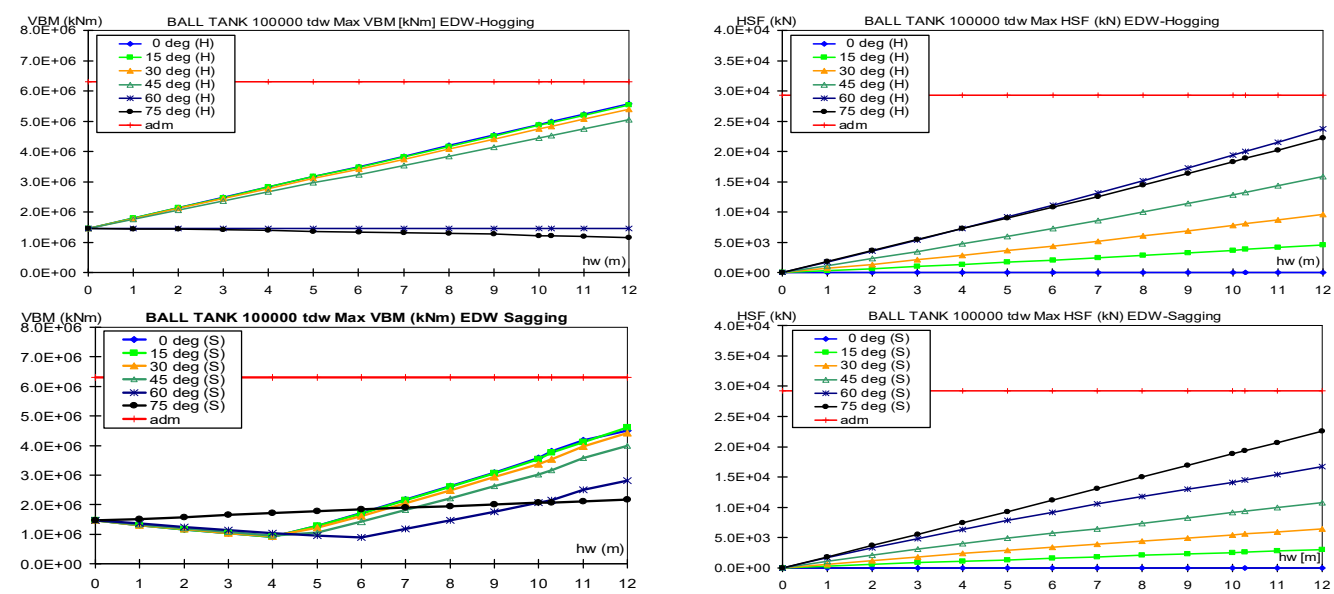

Fig.7.a $V B M_{\max }[\mathrm{kNm}]$, TK 100000, ballast
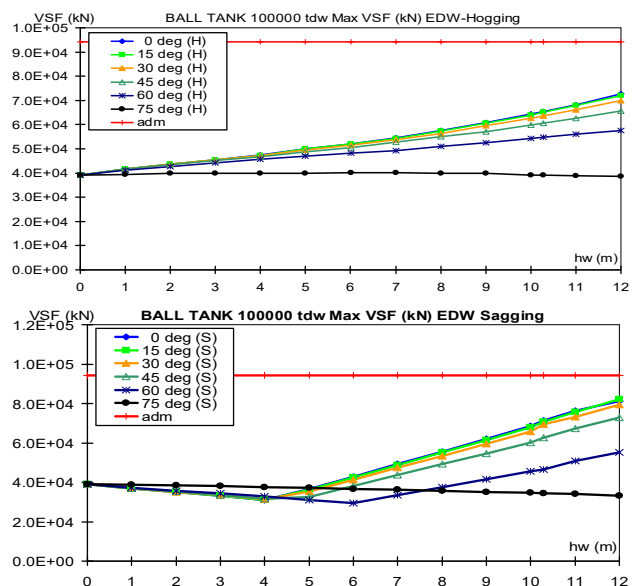

Fig.7.b $V S F_{\max }[\mathrm{kN}]$, TK 100000, ballast
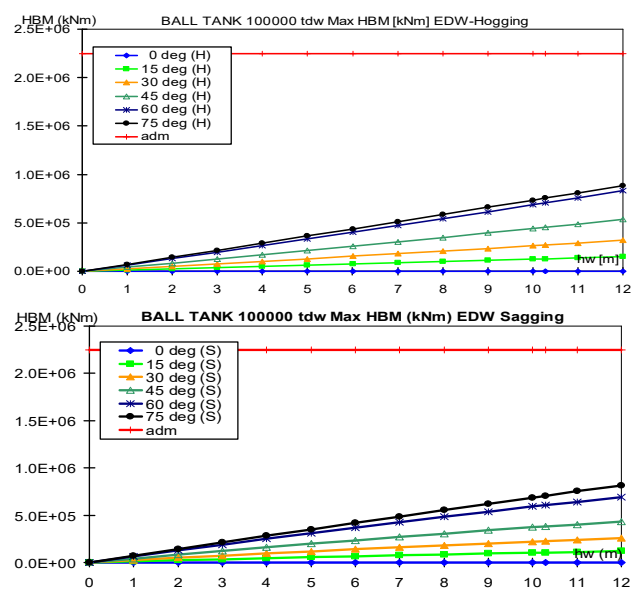

Fig.7.c $H B M_{\max }[\mathrm{kNm}]$, TK 100000, ballast

Fig.7.d $H S F_{\max }[\mathrm{kN}]$, TK 100000 , ballast

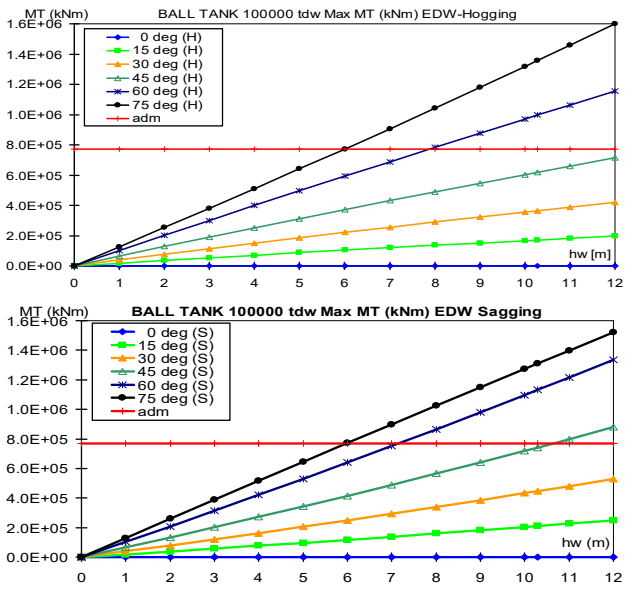

Fig.7.e $M T_{\max }[\mathrm{kNm}]$, TK 100000, ballast Zaft_PS (m) BALL TANK 100000 tdw Max Zaft_PS (m) EDW-Hogg \& Sagg

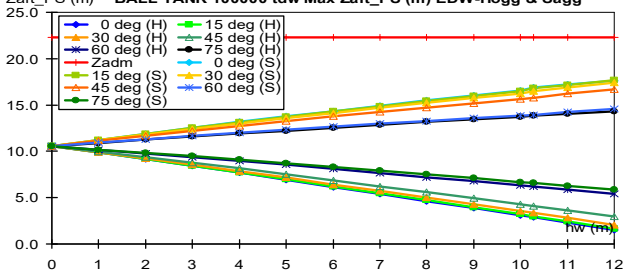

Zfore PS (m) BALL TANK 100000 tdw Max Zfore_PS (m) EDW-Hogg \& Sagg

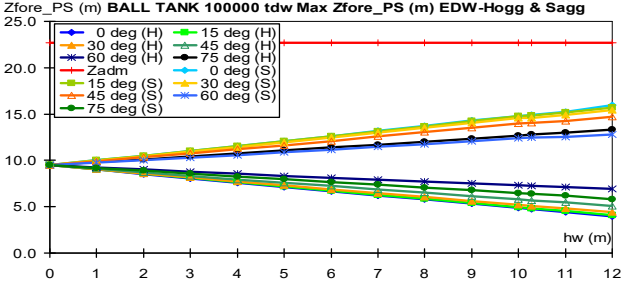

Fig.7.f $Z_{\max }[\mathrm{m}]$, aft-fore, TK 100000, ballast 


\section{CONCLUSIONS}

Fig. 8 and Table 7 present the resulting operation wave height $h_{w}$ limits, by the preliminary global strength criteria (Tab.2) for the 100000 tdw tanker [4] (Table 1, Fig.1).

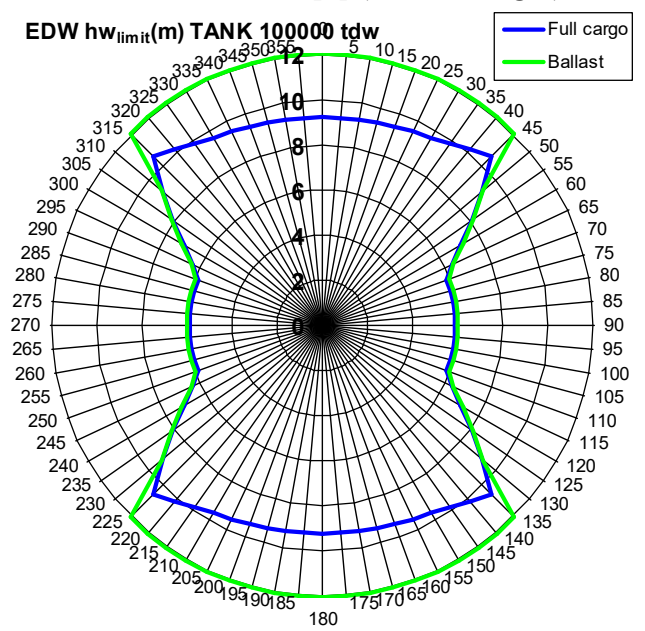

Fig.8 $h_{w}[\mathrm{~m}]$ limits, TK 100000, polar diagram

Table 7. $h_{w}[\mathrm{~m}]$ limits, TK 100000 , both cases \begin{tabular}{|l|l|l|l|l|l|l|}
\hline$\mu$ & $V B M$ & $V S F$ & $H B M$ & $H S F$ & $M T$ & $F B$ \\
\hline
\end{tabular} Full cargo case : Hogging oblique waves $90: \mathbf{R 4 ( 5 7 \% )}$ \begin{tabular}{|l|l|l|l|l|l|l|}
\hline 0 & $\mathbf{9 . 1 7 9}$ & $\mathbf{9 . 7 1 2}$ & 12.0 & 12.0 & 12.0 & 12.0 \\
\hline 15 & $\mathbf{9 . 2 6 8}$ & $\mathbf{9 . 8 0 7}$ & 12.0 & 12.0 & 12.0 & 12.0 \\
\hline
\end{tabular} \begin{tabular}{|l|l|l|l|l|l|l|}
\hline 15 & $\mathbf{9 . 2 6 8}$ & $\mathbf{9 . 8 0 7}$ & 12.0 & 12.0 & 12.0 & 12.0 \\
\hline 30 & $\mathbf{9 . 6 0 4}$ & $\mathbf{1 0 . 1 7 1}$ & 12.0 & 12.0 & 12.0 & 12.0 \\
\hline
\end{tabular} \begin{tabular}{|l|l|l|l|l|l|l|}
\hline 30 & $\mathbf{9 . 6 0 4}$ & $\mathbf{1 0 . 1 7 1}$ & 12.0 & 12.0 & 12.0 & 12.0 \\
\hline
\end{tabular}

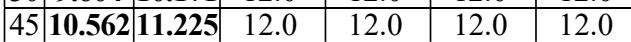
\begin{tabular}{|l|l|l|l|l|l|l|}
\hline 60 & 12.0 & 12.0 & 12.0 & $\mathbf{1 0 . 3 6 4}$ & $\mathbf{7 . 5 8 5}$ & 12.0 \\
\hline 75 & 12.0 & 12.0 & 12.0 & $\mathbf{1 1 . 3 6 0}$ & $\mathbf{5 . 8 5 4}$ & 12.0 \\
\hline
\end{tabular} \begin{tabular}{|l|l|l|l|l|l|l|}
\hline 75 & 12.0 & 12.0 & 12.0 & $\mathbf{1 1 . 3 6 0}$ & $\mathbf{5 . 8 5 4}$ & 12.0 \\
\hline
\end{tabular} Full cargo case : Sagging oblique waves 90:R4(57\%)

\begin{tabular}{|c|c|c|c|c|c|c|}
\hline 0 & 12.0 & 12.0 & 12.0 & 12.0 & 12.0 & 12.0 \\
\hline 15 & 12.0 & 12.0 & 12.0 & 12.0 & 12.0 & 12.0 \\
\hline 30 & 12.0 & 12.0 & 12.0 & 12.0 & 12.0 & 12.0 \\
\hline 45 & 12.0 & 12.0 & 12.0 & 12.0 & $\mathbf{1 0 . 5 5 4}$ & 12.0 \\
\hline 60 & 12.0 & 12.0 & 12.0 & 12.0 & $\mathbf{7 . 0 1 9}$ & 12.0 \\
\hline 75 & 12.0 & 12.0 & 12.0 & $\mathbf{1 0 . 5 0 6}$ & $\mathbf{5 . 8 3 0}$ & 12.0 \\
\hline
\end{tabular}
Ballast case : Hogging oblique waves $90: \mathbf{R 4 ( 5 8 \% )}$ \begin{tabular}{l|l|l|l|l|l|l|}
\hline 0 & 12.0 & 12.0 & 12.0 & 12.0 & 12.0 & 12.0 \\
\hline 15 & 12.0 & 12.0 & 12.0 & 12.0 & 12.0 & 12.0 \\
\hline
\end{tabular}

\begin{tabular}{|l|l|l|l|l|l|l|}
\hline 15 & 12.0 & 12.0 & 12.0 & 12.0 & 12.0 & 12.0 \\
\hline 30 & 12.0 & 12.0 & 12.0 & 12.0 & 12.0 & 12.0 \\
\hline
\end{tabular}

\begin{tabular}{|l|l|l|l|l|l|l|}
\hline 45 & 12.0 & 12.0 & 12.0 & 12.0 & 12.0 & 12.0 \\
\hline 60 & 12.0 & 12.0 & 12.0 & 12.0 & $\mathbf{7 . 8 7 2}$ & 12.0 \\
\hline
\end{tabular}

\begin{tabular}{|l|l|l|l|l|l|l|}
60 & 12.0 & 12.0 & 12.0 & 12.0 & $\mathbf{7 . 8 7 2}$ & 12.0 \\
\hline 75 & 12.0 & 12.0 & 12.0 & 12.0 & $\mathbf{5 . 9 7 5}$ & 12.0 \\
\hline
\end{tabular}

Ballast case : Sagging oblique waves $90: \mathbf{R 4 ( 5 8 \% )}$

\begin{tabular}{|c|c|c|c|c|c|c|}
\hline 0 & 12.0 & 12.0 & 12.0 & 12.0 & 12.0 & 12.0 \\
\hline 15 & 12.0 & 12.0 & 12.0 & 12.0 & 12.0 & 12.0 \\
\hline 30 & 12.0 & 12.0 & 12.0 & 12.0 & 12.0 & 12.0 \\
\hline 45 & 12.0 & 12.0 & 12.0 & 12.0 & 12.0 & 12.0 \\
\hline 60 & 12.0 & 12.0 & 12.0 & 12.0 & $\mathbf{7 . 1 5 5}$ & 12.0 \\
\hline 75 & 12.0 & 12.0 & 12.0 & 12.0 & $\mathbf{5 . 9 8 3}$ & 12.0 \\
\hline
\end{tabular}

From the numerical data in sections 2 and 3 the next conclusions are formulated: 1. The wave height \& ship-wave angle, combined with the geometric nonlinearities of the 1000 tdw tanker hull shape (Fig.1), lead to significant changes of the equilibrium parameters (sinkage, pitch, roll) (Tables 3.a,b \& 5.a,b, Figs. 2.a,b, Figs.5.a,b) and also for the sectional efforts values (Figs.3.a-e, Figs.6.a-e).

2 . In the case of full cargo, the main global strength restrictions occur in hogging wave condition (Figs.4.a-e, Table 4.a,b), for the heading angle whole range, and in sagging wave condition for $\mu>30 \mathrm{deg}$.

3. In the case of ballast, the global strength restrictions are similar in hogging and sagging conditions and occur for heading angle $\mu>45$ deg (Figs.7.a-e, Table 6.a,b).

4. For both loading cases the free board criteria has no restriction (Fig.4.f, Fig.7.f).

5. The beam sea state $(\mu=75-105 \mathrm{deg}$.) is the most restrictive navigation case, $h_{\text {wlimit }}=5.830$ -5.975 m, R4(57-58\%) [1] (Table 7, Fig.8).

6. Extended studies shall be coupled with $3 \mathrm{D}$ models and other relevant evaluation criteria.

\section{Acknowledgements}

This study and the program P_QSW [2] are developed at the Naval Architecture Research Centre, from University "Dunarea de Jos" of Galati.

\section{REFERENCES}

[1]. DNV-GL, “Rules. Maritime Ships”, Det Norske Veritas - Germanischer Lloyd, 2020.

[2]. Domnisoru, L.," Special chapters on ships' structures analysis. Applications", "Dunarea de Jos" University Foundation Publishing House, Galati, 2017.

[3]. Domnisoru, L., "On operation capabilities analysis of a 100000 tdw tanker by seakeeping criteria",The Annals of "Dunarea de Jos" Univ. Galati, Fascicle XI Shipbuilding, pp.31-40, 2019.

[4]. Dumitru, D., "Compendium of ships forms", "Dunarea de Jos" University Foundation Publishing House, Galati, 2014.

[5]. ISSC, "Proceeding of the 20th ISSC Congress", IOS Press, Amsterdam, 2018.

Paper received on October $16^{\text {st }}, 2020$

(C) Galati University Press, 2020 